\title{
A Quality Platform for Sharing Information on Nutrition and Metabolism
}

With the first issue of volume 58 (2011), the editorial office of the Annals of Nutrition and Metabolism has moved from Vienna to Munich. It is a great honour and responsibility to serve as the new Editor of this renowned journal, which has a history of more than 50 years and a very strong track record. Based on considerable experience gathered as editorial board member and associate editor of other journals, I hope to be able to master this enormous challenge and do look forward to this notable task. My sincere thanks go to the previous editor, Prof. Ibrahim Elmadfa, who has managed the journal extremely well over more than a decade, and now hands over a healthy journal in a strong position. The current impact factor now is 1.970 , and the 5 -year impact factor is 2.023 . I greatly appreciate the advice Prof. Ibrahim Elmadfa has provided to me and hope to benefit from his continued support.

From volume 58 onwards, the Editor will be assisted by a strong team of Associate Editors with highly qualified and experienced researchers, including Angel Gil (Granada), Lisette de Groot (Wageningen), Gernot Desoye (Graz), Joachim Heinrich (Munich), Lucilla Poston (London), Matthias Schulze (Potsdam) and Michael Zimmermann (Zürich; introduced on page IV). I am very grateful indeed to all of them for kindly accepting the invitation to join. In addition, a strong editorial board supports the journal and provides critical input. Birgit Kessler (Munich) serves as the editorial assistant.

Our common goal is to maintain and further strengthen the role of the Annals of Nutrition and Metabolism as a leading international peer-reviewed journal for sharing information on human nutrition, metabolism and related fields, covering the broad and multidisciplinary nature of science in nutrition and metabolism. As the official journal of both the International Union of Nutritional Sciences (IUNS) and the Federation of European Nutrition Societies (FENS), the journal has a high visibility among both researchers and users of research results, including policy makers, across Europe and around the world. We aim at attracting some of the best manuscripts from all areas relevant to human nutrition and metabolism. Therefore, together with S. Karger Publishers we strive for a rapid peer review of submitted manuscripts and fast online publication of accepted papers.

The scope of the journal covers basic science as it relates to human nutrition and metabolism, including cell biology, molecular and genetic approaches; experimental models; methodological issues; observational and intervention studies; papers on foods; diets and dietary supplements; energy metabolism; macro- and micronutrients including vitamins and minerals; bioactive components; dietetics; obesity; clinical nutrition; health prevention; social sciences and health economy; policy issues; etc. In addition to 'Original Papers', the journal will publish 'Review Articles' on pertinent topics, 'Systematic Reviews', short 'Commentaries and Viewpoint' articles that can address hot or controversial questions, short 'Meeting Reports', 'Letters to the editor', and 'Announcements/Society News'. The journal will also publish 'Supplements' with proceedings from internationally relevant conferences on nutrition and metabolism.

In order to best serve the scientific community, our authors and our readers, we would love to hear your comments and suggestions. Please do not hesitate to share your observations with us.

$$
\begin{aligned}
& \text { Prof. Berthold Koletzko, Editor } \\
& \text { University of Munich Medical Centre }
\end{aligned}
$$

\section{KARGER}

두 2011 S. Karger AG, Basel

Fax +41613061234

E-Mail karger@karger.ch

www.karger.com 


\section{Nutrition\& Metabolism}

\section{Introduction of the New Editors of Annals of Nutrition and Metabolism}

\section{Editor-in-Chief}

\section{Berthold Koletzko}

Berthold Koletzko is a Professor of Pediatrics and Head of the Division of Metabolic and Nutritional Medicine at Dr. von Hauner Children's Hospital, University of $\mathrm{Mu}-$ nich Medical Center, Munich, Germany. He is the author of more than 750 publications and has coordinated national and international research projects including the Euro-

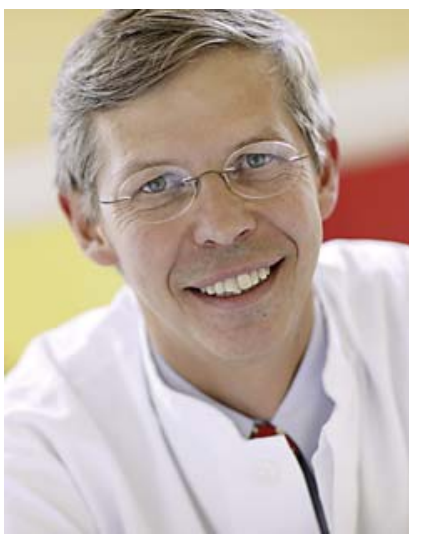
pean Childhood Obesity Project, the Early Nutrition Programming Project, the Multidisciplinary Early Modification of Obesity Risk Project, and the Research Network on Malnutrition and Outcome in Hospitalized Children in Europe. Scientific leadership roles include Chair of the Committee on Nutrition of the German Society of Pediatrics, Chair of the Child Health Foundation, Scientific Advisor to the Committee on Environment, Health, and Consumer Protection of the European Parliament, Scientific Advisor to the Forum on Early Childhood Advancement of the German Federal Family Minister, Managing Council of the National Platform on Nutrition and Physical Activity, Chair of the Scientific Board of the Federal Government Network for Young Families, and President of the German Society for Nutritional Medicine.

\section{Associate Editors}

\section{Lisette C.P.G.M. de Groot}

Lisette de Groot is a Professor of Nutrition and Ageing at the Division of Human Nutrition at Wageningen University, the Netherlands. She was trained in nutrition, physiology, and epidemiology at Wageningen University, where she finished her MSc in nutrition in 1984 and her PhD in physiology in 1988. With academic posts at several univer-

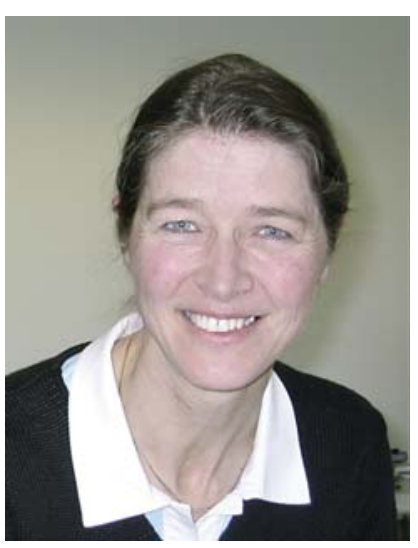
sity departments she has gained 20 years of research experience in the field of the nutrition and health of elderly people, both in nutritional epidemiology [including SENECA (Survey in Europe on Nutrition and the Elderly) and EURRECA (European Micronutrient Recommendations Aligned)] and in intervention studies in old age. Her research interests center around the role of nutrition in the maintenance of health in old age, with due attention to nutritional ways to slow down ageing-related functional decline. So far, she has published over 150 scientific papers. 


\section{Gernot Desoye}

Gernot Desoye is a Professor of Biochemistry and Director of Research, Department of Obstetrics and Gynecology of the Medical University of Graz, Graz, Austria. He leads a research team fo-

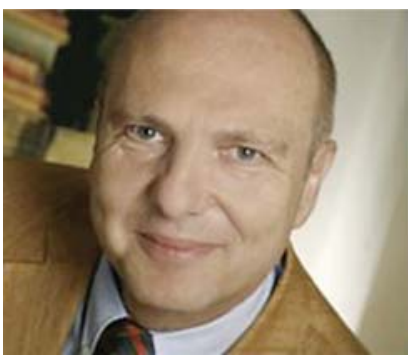
cusing on the effects of maternal nutrition, obesity, and diabetes on placental development and function and how this affects the fetus. Currently, he coordinates the FP7-funded project DALI and has long-standing experience in worldwide collaborative studies beyond the collective efforts of a recent EC-funded project on nutrient effects in perinatology (PeriLip). He has managed several international research projects and is a member of many international societies and of several executive boards including Diabetic Pregnancy Study Groups in Europe (DPSG) and USA-West, past chair of DPSG, recipient of the Jorgen Pedersen Award 2008 of DPSG, emeritus editor in chief of Placenta, past editorial board member of Trophoblast Research, and ad hoc reviewer for more than 30 international journals and granting bodies. He has been evaluator and auditor for the EC (FP5FP7) and was recently invited as an expert on diabetes in pregnancy to the European Parliament.

\section{Joachim Heinrich}

Joachim Heinrich is an epidemiologist and Deputy Director of the Institute of Epidemiology at Helmholtzzentrum München, the German Research Center for Environmental Health. He has authored more than 500 publications and has been involved in numerous national and international epidemiological research projects examining

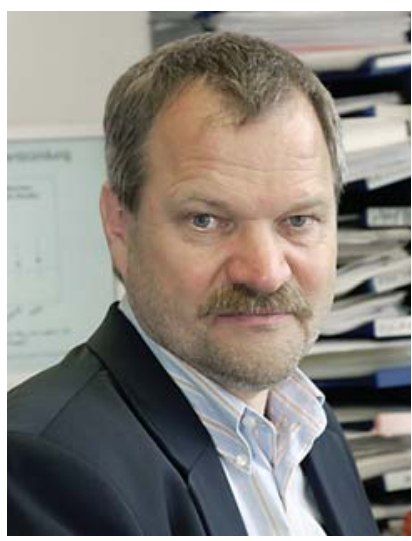
a broad spectrum of common chronic diseases, specifically the relationship between diet, genetics, and respiratory health.

\section{Angel Gil}

Angel Gil is a Professor of Biochemistry and Molecular Biology at the University of Granada, Spain. He has authored more than 320 peer-reviewed articles and more than 75 book chapters. He managed the R\&D team of Puleva SA and Abbott Lab for 13 years and developed dietetic foods. Until 2004, he was Director of the University of

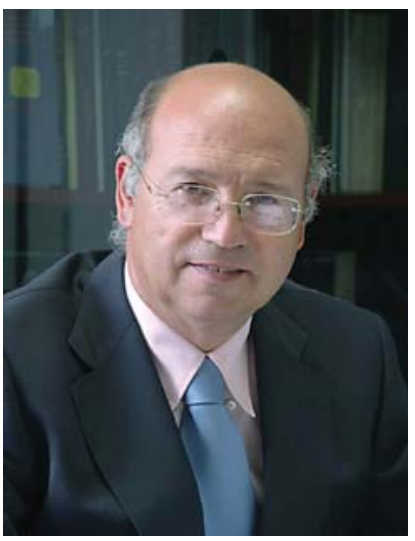
Granada Foundation. His research comprises the biological effects of dietary nucleotides, lipid metabolism in early life, the effects of olive oil on cardiovascular health, and nutrigenomics, particularly the gene expression of adipose and muscle tissue in obesity and the relation to endocrine and metabolic changes. He is President of the Iberoamerican Council for Nutrition Journals, Vice President of the Spanish Society of Nutrition, and member of the Scientific Committee of the Spanish Society of Parenteral and Enteral Nutrition, the Spanish Academy of Nutrition, and the Royal Academy of Veterinary Sciences. He is President of the IUNS International Congress of Nutrition 2013.

\section{Lucilla Poston}

Professor Lucilla Poston is Head of the Division of Women's Health and Director of the Maternal and Fetal Research Unit at the School of Medicine of King's College London. Professor Poston graduated in physiology from University College London and undertook her $\mathrm{PhD}$ at King's College London. She was appointed to her present

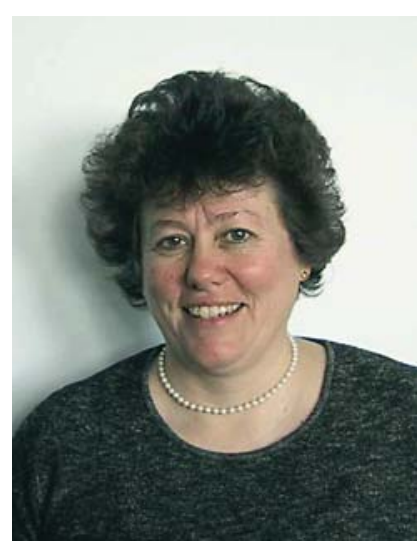
position as Tommy's the Baby Charity Chair of Maternal and Fetal Health in 2005. Her particular research interests include nutrition in pregnancy, preeclampsia and developmental origins of disease. Professor Poston is an honorary Fellow of the Royal College of Obstetricians and Gynecologists, an NIHR Senior Investigator (UK), and Fellow of the Academy of Medical Sciences (UK). She has published more than 200 original research papers. 


\section{Matthias Schulze}

Matthias Schulze studied Nutritional Sciences at Friedrich Schiller University Jena, Germany, and Public Health at Tulane University, New Orleans. He got his $\mathrm{PhD}$ training (Dr. $\mathrm{PH})$ at Technical University Berlin in cooperation with the German Institute of Human Nutrition (DIfE). After postdoctoral work at Harvard

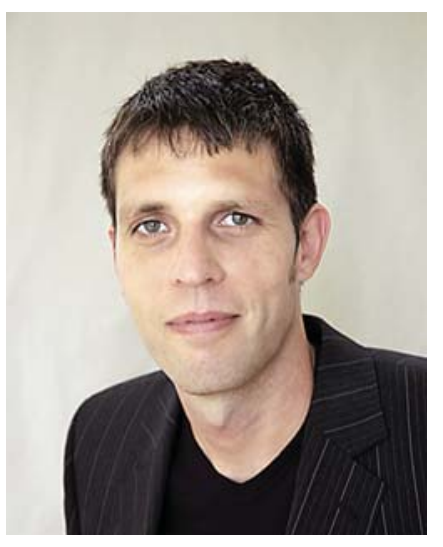

School of Public Health (2002-2004) and DIfE (20042008), he became a Professor at Technical University Munich and Head of the Public Health Nutrition Unit in 2008. Matthias Schulze was appointed as full Professor at the University Potsdam and Head of the Department of Molecular Epidemiology at DIfE in 2010. His research interests are methodological approaches for dietary pattern analyses, metabolic and genetic predictors of type 2 diabetes, interaction between genetic factors and diet in the development of type 2 diabetes, and risk prediction modeling.

\section{Michael Zimmermann}

Michael Zimmermann received his MD from Vanderbilt University School of Medicine, where he was President of the AOA Medical Honor Society. He completed his postgraduate medical training at the University of California in San Francisco and was a postdoctoral fellow at the University of California at

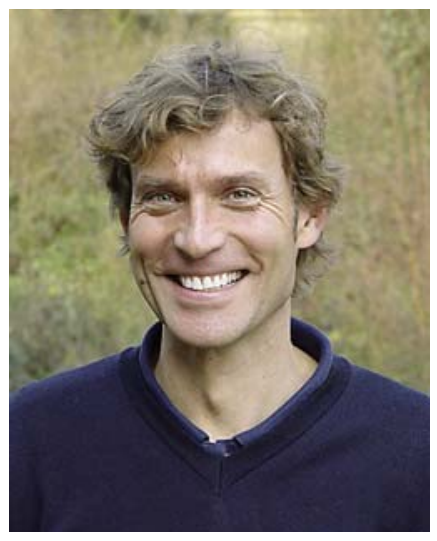
Berkeley, where he received an MSc in nutritional science. $\mathrm{He}$ is currently Senior Scientist in the Laboratory for Human Nutrition at the Swiss Federal Institute of Technology in Zürich (ETHZ). He is also a Professor at Wageningen University in the Netherlands, where he holds the Unilever Endowed Chair in International Health and Micronutrients. His research focuses on nutrition and metabolism, including micronutrient deficiencies. 